\title{
La prolongation des conflits : Approche comparative des systèmes de guerre
}

Table ronde

\section{(2) OpenEdition}

1 Journals

\section{Édition électronique}

URL : http://journals.openedition.org/conflits/147

DOI : $10.4000 /$ conflits. 147

ISSN : $1777-5345$

Éditeur :

CCLS - Centre d'études sur les conflits lilberté et sécurité, L'Harmattan

Édition imprimée

Date de publication : 21 janvier 1990

ISSN : 1157-996X

Référence électronique

Table ronde, «La prolongation des conflits: Approche comparative des systèmes de guerre », Cultures \& Conflits [En ligne], 01 | hiver 1990, mis en ligne le 01 février 2005, consulté le 30 mars 2021. URL:

http://journals.openedition.org/conflits/147 ; DOI : https://doi.org/10.4000/conflits.147

Ce document a été généré automatiquement le 30 mars 2021.

Creative Commons License 


\title{
La prolongation des conflits : Approche comparative des systèmes de guerre
}

\author{
Table ronde
}

1 Didier Bigo : A titre d'introduction à cette table ronde $1^{1}, \mathrm{j}^{\prime}$ 'aimerais rappeler les idées qui ont prévalu au choix de ce thème de la prolongation des conflits pour ce premier numéro. Il s'agissait tout d'abord d'illustrer, à travers ce choix de "conflits qui durent", l'originalité du projet qui nous réunit, à savoir : mieux comprendre les enracinements sociétaux des conflits dits locaux, voir que ces conflits, qui peuvent dans certaines occasions bouleverser les relations internationales, ont des causes internes puissantes, produisent des dynamiques sociales qui recomposent les sociétés et les cultures qui leur ont donné naissance, et constituent des phénomènes dont l'explication n'est jamais réductible, ni aux "politiques étrangères" des États, ni même à leurs géopolitiques, ou au degré de stabilité de "l'ordre international".

2 Située à l'interface de la discipline des "relations internationales", des "questions de stratégie" et de "l'analyse comparée des systèmes politiques", l'étude des conflits a longtemps souffert d'un écartèlement qui conduit à autant de réductionnismes et de simplifications outrancières. Il me semble donc nécessaire, afin de promouvoir une analyse comparée des sociétés politiques et des conflits, de créer un lieu où puissent se retrouver anthropologues qui s'intéressent au contemporain, historiens, sociologues, politistes spécialisés dans des aires régionales différentes et politistes s'occupant de relations internationales pour que puissent émerger des problématiques suffisamment fines pour rendre compte de la complexité des formes et des dynamiques conflictuelles, surtout à un moment où tout le monde reconnait qu'elles conditionnent notre avenir et ne sont pas un simple épiphénomène de la situation de "paix, ni guerre" décrite par Raymond Aron. A cet égard, ce numéro me semble une concrétisation de cet effort, mais il faudrait éviter qu'il ne se résume à une série de monographies où les textes, quelle que soit leur pertinence, ne dialogueraient pas entre eux et ne chercheraient pas à échapper aux cloisonnements des aires géopolitiques dont le culturalisme inconscient 
a toujours empêché d'analyser les répertoires politiques et culturels des sociétés en conflit. J'espère justement que notre refus d'une géopolitique, où la contiguïté territoriale fait office de problématique, nous permettra une approche des cultures politiques évitant les pièges du culturalisme et des cloisonnements disciplinaires par aire géographique que l'Université ne cesse de reproduire. Les comparaisons qui émergeront de la table ronde permettront peut-être de poser les bases d'un tel projet et je vous remercie tous de votre participation.

3 Mais il est vrai qu'il y a aussi une raison plus conjoncturelle et plus polémique au choix de ce thème de la prolongation des conflits, celle de couper court à certaines idéologies qui se développent "l'ombre" des transformations de l'ordre international. Discours sur "la menace que ferait peser le Sud sur la sécurité du Nord", dont nous traiterons dans le prochain numéro, et idéologie d'un désintérêt pour les conflits locaux au nom d'une vision du monde cartiériste et égocentrique qui confond la paix au Nord avec la paix dans le monde. Cette dernière idéologie a partie liée avec un certain nombre de lectures dominantes qui tiraient mécaniquement de la fin de l'antagonisme Est/Ouest que les conflits locaux allaient disparaître comme par magie. En effet, les lectures géostratégiques, influencées par l'école globaliste et par la surestimation de la valeur de la dissuasion nucléaire, faisaient des conflits régionaux des pays dits du Sud des conséquences des stratégies indirectes de l'affrontement Est/Ouest. Dans l'impossibilité de s'affronter sur le théâtre européen, les grandes puissances auraient de part et d'autre à envisager des stratégies de dérivation, d'encerclement... Chaque conflit local aurait été attisé par la recherche d'un avantage tactique sur le plan mondial obligeant l'autre puissance à se positionner dans le camp local adverse pour maintenir un "pat stratégique". Le couplage aurait permis ensuite aux grandes puissances de réguler les conflits au mieux de leurs intérêts, les attisant ou les limitant selon les périodes. Toute cette vulgate est connue, je n'insiste pas. Depuis longtemps déjà, des critiques ont été émises à l'égard de ce type d'analyse : les acteurs locaux ont leur propre autonomie, ils jouent eux-mêmes de la rivalité Est/Ouest pour créer un déséquilibre du rapport de forces grâce à des appuis extérieurs... Le couplage n'implique pas une mise en dépendance totale des acteurs locaux dans la conduite des opérations de guerre... Le débat restait néanmoins ouvert et très majoritairement, dans les médias au moins, la première thèse continuait à prévaloir. Comme on a lu à travers cette thèse les situations d'Afrique australe, d'Amérique centrale, de la corne de l'Afrique, d'Afghanistan... on croyait forcément que le rapprochement USA/URSS allait clore ces conflits. Seulement, cette idéologie de la pacification par le retrait des grandes puissances n'a pas résisté longtemps à la réalité du découplage entre les conflits régionaux et l'antagonisme Est/Ouest. Dès lors, incapable de comprendre pourquoi les conflits se prolongeaient, elle s'est convertie brutalement en une idéologie de la menace du Sud reproduisant jusqu'à la caricature celle de la menace de l'Est en pleine période MacCarthy . Nous y reviendrons dans le prochain numéro. Pour l'heure, il importe d'analyser les raisons de la prolongation des conflits en relation avec cette mutation de l'ordre international sans ramener les premiers à une péripétie de la seconde.

Rémy Leveau : tu as raison, cet effondrement du communisme qui a modifié à la fois les sources de légitimation et la structure de l'ordre bipolaire transforme profondément non pas tant la nature des conflits que le sens qu'on leur donne. Mais ce deuxième élément change nos grilles d'interprétation. Cela tient à la transformation de l'ordre international lui-même. Que peut signifier par exemple la notion de tiers monde 
quand la distinction entre le premier et le second fait de moins en moins sens? Que peut signifier à l'heure actuelle pour un guérillero d'utiliser un langage marxiste?

Zaki Laïdi : Oui. On a assisté avec la fin des idéologies révolutionnaires à la perte du sens des conflits qui les rendait négociables, monnayables à l'échelle internationale. L'évolution des mentalités est considérable et, dans le fond, les grands n'ont pas tout à fait tort lorsqu'ils disent que les conflits locaux ne les intéressent plus. Ils ne parlent plus le même langage, ils disparaissent de l'horizon politico-stratégique, cessent d'être visibles et peuvent donc se prolonger très longtemps sans produire le moindre effet sur la scène internationale.

Daniel Hermant : C'est là l'idéologie dont parlait Didier tout à l'heure. Il me semble d'ailleurs que nous sommes tous d'accord pour refuser ces approches mutilantes qui réduisent chacun des conflits aux "séquelles de l'antagonisme Est/Ouest", pour reprendre la formule du texte d'Olivier Roy.

7 Didier Bigo : Que ce soit Olivier Roy, Olivier Dabène, François Guilbert ou Philippe Chapleau, il y a une même préoccupation méthodologique: aller à l'encontre du globalisme et montrer les dimensions originales et irréductibles de chaque conflit. On ne peut pas tirer du retrait soviétique d'Afghanistan, des élections au Nicaragua consacrant la chute des sandinistes, de l'accord de paix du 28 août 1990 sous la tutelle de l'ONU, du retrait conjoint des Cubains et des Sud-Africains d'Afrique australe que les conflits vont s'arrêter; ils ne font que se métamorphoser en diminuant d'intensité et en se désenchevêtrant partiellement par perte du niveau le plus englobant, celui qui permettait leur convertibilité à l'échelle idéologique mondiale pour reprendre la métaphore de Zaki Laïdi.

8 Au-delà de ce débat sur le rapport à l'ordre international, il faut sans doute revenir sur les raisons profondes de la prolongation des conflits. Alain Gascon nous invite, au-delà des enjeux géostratégiques du moment, à ré-enraciner les conflits dans le temps long braudélien, à étudier l'impact des constructions historiques légitimatoires sur les stratégies des acteurs, et à voir quelles sont leurs ré-interprétations de l'Histoire en fonction des différents répertoires que leur histoire politique leur fournit, ce qui me semble une piste intéressante. Salim Nasr, lui, insiste sur un point clé. Si l'Histoire est importante, il ne faut pas tomber dans une illusion étiologique expliquant les conflits par l'actualisation de potentialités latentes d'antagonismes sociaux entre communautés ou entre groupes, il faut analyser le processus même du conflit, sa dynamique propre. C'est sur ce point sans doute qu'émerge une problématique commune malgré la variété des traitements, la diversité des objets, la pluralité des disciplines convoquées pour l'explication. On peut parler d'un "système de guerre" : ou d'une "société de guerre" qui émerge du conflit et qu'ensuite produit les conditions de sa reproduction.

Ce système de guerre a des acteurs qui lui sont propres, les acteurs militarisés qui entretiennent entre eux des liens équivoques et qui dominent les tiers du conflit, les populations civiles, les acteurs désarmés. Il génère de nouveaux fronts et frontières qui ont une double fonction, délimiter les zones de conflit (les circonscrire, les forclore) et surtout imposer un ordre sociopolitique aux populations désarmées en les soumettant aux différents chefs de guerre. Ce système se légitime par la poursuite de la guerre mais paradoxalement, plus il se prolonge, plus les buts de guerre sont oubliés et plus une connivence objective entre les acteurs militarisés apparaît. Cela s'explique par deux traits principaux : la restructuration de l'économie productive en faveur des chefs de guerre avec la mise en place de douanes, de taxes, bref, d'un racket économique, d'une 
économie mafieuse autour des fronts : les chefs de guerre s'appropriant les ressources extérieures et les redistribuant selon des schémas clientélistes. Il y a là une véritable économie parasitaire qui vit de son interposition sur les flux économiques. Le deuxième trait tient à la dynamique conflictuelle elle-même. Plus le conflit se prolonge, plus, semble-t-il, il y a perte des motivations idéologiques, instrumentalisation des alliances, professionnalisation des acteurs militarisés et socialisation par la guerre (un âge plus jeune au combat, des groupes sociaux plus défavorisés...). On ne cherche plus à conquérir le territoire adverse, mais simplement à conserver sa zone et à faire quelques incursions pour piller les biens adverses, légitimant du même coup sa fonction de domination. En effet, au fur et à mesure du déroulement du processus conflictuel, il devient plus important d'obtenir l'obéissance sans faille des civils désarmés dans sa zone de contrôle que de lutter contre l'adversaire. Celle-ci n'est plus, à la limite, que la légitimation de la domination sur les tiers. Finalement peut apparaître, comme dans le cas libanais, une connivence objective entre les acteurs militarisés. On passe donc de la guerre contre l'adversaire à la complicité entre acteurs militarisés pour maintenir une guerre "homéopathique" dont la fonction principale est de légitimer la domination sur la zone de contrôle.

10 Zaki Laïdi : Le cas du Polisario est tout à fait caractéristique de ce type de logique que tu décris. Il y a eu création d'un système autoritaire sur les Sahraouis, un peu de même nature que celui imposé par l'OLP sur les Palestiniens. Fn effet, pour le Polisario, la guerre est le seul moyen d'assurer sa survie. Mais il ne faut pas généraliser trop vite, il existe des trajectoires très différentes.

11 Didier Bigo : Oui, je n'ai pas voulu présenter ici un modèle unique qui rendrait compte des évolutions de chaque cas, mais simplement tracer à grands traits les éléments de problématiques qui permettent de comparer les conflits, autant pour rendre compte de leurs différences de trajectoires que pour montrer leurs traits communs. Loin de vouloir enserrer dans un cadre unique réducteur, il me semble important de dégager des dynamiques différenciées qui rendent compte des trajectoires suivies. On ne pourra le faire qu'à la condition de savoir quoi comparer, et c'est là peut-être que l'ouvrage de Christian Geffray, les remarques de Salim Nasr, d'Olivier Roy et de Guy Nicolas dont je me suis inspiré ont leurs valeurs, au-delà des différents cas d'espèces.

12 François Guilbert : Au Cambodge, on peut peut-être parler de système de guerre. Les parties en lutte fondent leur légitimité sur le contrôle des populations et des camps de réfugiés. Les groupes de guérilla obligent les populations civiles à revenir de force dans les zones dites libérées et se battent pour les contrôles des camps et de l'aide humanitaire. Il n'y a pas pour autant de connivence entre les adversaires. Les Khmers rouges ont la volonté d'abattre le gouvernement de Phnom Penh, même s'ils préfèrent une guerre de position à des attaques frontales.

13 Alain Gascon : Connivence est peut-être exagéré ; il existe des conflits bien réels entre les groupes de guérilla et le pouvoir ainsi qu'à l'intérieur de chaque groupe. En revanche, chacun s'installe dans la guerre et cherche surtout à maintenir son autorité sur les populations. Le FPLE développe un encadrement des populations, se veut un "quasi-État" sur sa zone, construit des écoles, des hôpitaux.

Olivier Roy: En Afghanistan, la violence est plus démonstrative que réelle; il y a autolimitation de la violence. Chaque groupe maintient ses positions sur son territoire et dépasse rarement les limites géographiques de son quawm. Une société de guerre 
s'installe parce que les "nouveaux chefs" tirent leurs pouvoirs de la circulation des biens induite par la guerre.

Olivier Dabène : A l'échelon local, au Guatemala par exemple, on pourrait aussi parler de "système de guerre". Il y a des acteurs militarisés et une population asservie, mais il $\mathrm{y}$ a aussi un système de répression dont jusqu'ici nous n'avons pas parlé et qui explique pour beaucoup les dynamiques conflictuelles et la prolongation des conflits. La notion de "système" ne doit pas non plus faire oublier qu'il y a des entrées et des sorties du système. L'Amérique centrale semble réussir malgré tout à sortir des conflits. Ce qui n'empêche pas des économies de gang de se développer.

François Guilbert: Les groupes de guérilla ont de plus en plus besoin d'avoir des financements importants; ils constituent des structures quasi étatiques. Comme l'évolution des rapports Est/Ouest leur retire leur rente de situation, ils se débrouillent autrement, à travers des trafics de pierres précieuses, d'armements et de drogue. D'ailleurs, au Cambodge, l'État n'a guère plus de ressources que les mouvements d'opposition. Même pour les ressources extérieures, il est concurrencé. Il y a un véritable marché de l'aide humanitaire qui est. En train de se mettre en place, et chaque groupe sort maintenant des plaquettes soignées pour expliquer ses besoins et non plus des feuilles ronéotées. Le militantisme en chute libre est remplacé par de véritables organes techniques de promotion internationale.

Roland Marchal: Jusqu'à présent, nous n'avons pas encore évoqué un point fondamental, celui des diasporas et de l'aide humanitaire qui pourtant contribuent pour beaucoup à la prolongation des conflits. Dans la corne de l'Afrique, les mouvements érythréens se financent par leur intermédiaire. L'aide humanitaire n'est pas forcément détournée, mais les mouvements qui assurent son transport sur place prélèvent une sorte de dîme qui leur permet un financement de leurs activités. On retrouverait la même chose pour l'Afghanistan, le Cambodge...

François Guilbert : Oui. L'aide humanitaire est très importante dans la mesure où elle a rendu visibles des conflits régionaux pendant très longtemps, et a assuré des flux d'argent considérables. On retrouve ici les problèmes de financement des mouvements qui entraînent une logique plus "économique" que "militaire", comme celle que les Khmers rouges sont en train de mettre sur pied avec les trafics de pierres précieuses et la recherche d'un accès à la mer. Problèmes qui débordent sur la Thaïlande...

Rémy Leveau : L'exemple libanais est un indicateur de ce qui pourrait se passer si ces logiques mafieuses se développaient. L'alternative peut être formulée de la manière suivante : économie de gang ou retour au politique? Dans la mesure où le maintien d'un véritable gouvernement libanais semble irréaliste, les Syriens qui contrôlent le Liban sont devant le dilemme suivant : soit on fait peser une lourde tutelle sur le Liban, mais on s'expose à une fuite des capacités qui transformerait le pays en zone grise sans dynamisme ; soit on laisse une liberté relative au Liban qui lui permettra de continuer à être un centre économique lié aux circuits mondiaux. On se trouverait alors dans une situation qui ne serait pas sans rappeler le Nigeria décrit par Guy Nicolas. Une espèce d'ordre fédéral (sous contrôle syrien) équilibrant des forces contraires. La Syrie pourrait être le garant d'un ordre régional exerçant un pouvoir d'arbitrage sur un HongKong local.

Des diverses hypothèses que l'on faisait lors de la fin de la confrontation Est/Ouest, la plus vraisemblable après une année n'est probablement pas l'hypothèse optimiste: celle d'une réduction des conflits provoquée par la fin de la rivalité Est/Ouest et qui se 
prolongerait par les progrès de la démocratie et ceux de l'économie de marché. On doit plutôt envisager l'hypothèse la plus pessimiste, celle de la possibilité d'une marginalisation de très vastes secteurs de la population du tiers monde appuyés sur une économie délinquante (au Liban, en Amérique centrale, mais pourquoi pas demain au Maghreb et en Afrique subsaharienne), dirigés par des groupes militaro-politiques ou des groupes idéologico-religieux ; avec peut-être quelques secteurs qui resteront liés à l'économie internationale. Le texte de Guy Nicolas envisageant la résurrection d'une politique de comptoirs telle qu'elle a pu exister au XIXème siècle va dans le même sens. $\mathrm{Au}$ Nigeria, un vaste ensemble régional permet de ré-équilibrer des sous-ensembles violents. Les perspectives de ce type d'évolution ne sont pourtant pas réjouissantes. En effet, la dissémination de certains types d'armement, la prolifération éventuelle d'armes nucléaires feraient peser des contraintes inacceptables sur la communauté internationale.

21 Daniel Hermant: Nous reviendrons sur ce point dans le deuxième numéro, tout comme nous aborderons plus en détail les rapports mafia, drogue et politique dans le troisième.

Zaki Laïdi : Il ne faut pas pousser le raisonnement trop loin dans le sens d'une désagrégation des États-nations. En Angola, l'État sort, à mon avis, renforcé du conflit. $\mathrm{Au}$ Tchad, le niveau minimum correspond justement à la préservation de l'État-nation. Les groupes de guérilla ont toujours maintenu le cadre étatique. A mon sens, les conflits ne remettent pas en cause l'État.

Roland Marchal : Je n'en suis pas sûr. On assiste dans un certain nombre de cas à la fin de la contrainte étatique. L'évolution de l'Éthiopie semble se faire au détriment de l'unité ; elle va vers la dispersion.

24 Rémy Leveau: Cette désintégration étatique se fait sous le regard des puissances régionales. Les Égyptiens, toujours inquiets quand on touche aux sources du Nil, les Soudanais, les Arabes...

Didier Bigo : En Éthiopie comme en Somalie, il existe une atomisation de la violence marquée par la gestion concurrentielle des "fronts de libération"; on est dans des situations où personne ne peut gagner, où les alliances ont pour but d'empêcher un déséquilibre au bénéfice d'un mouvement. Le pouvoir n'a plus aucun attribut étatique, il pourrait être renversé par une conjonction d'alliances, mais chacun des mouvements s'y refuse.

Alain Gascon : Cela s'explique. L'Éthiopie est un vaste territoire, où la guerre n'est pas toujours présente. Les intérêts de chacun sont différents. On peut d'ailleurs se demander s'il n'est pas plus onéreux d'assurer la survie de certaines régions pour le pouvoir, plutôt que de faire la guerre.

Rémy Leveau : Ces problèmes d'intégration et de désintégration rappellent à un autre niveau des problèmes traditionnels de science politique. Ne retrouve-t-on pas une situation du XIXème siècle, celle que décrit J. Chevalier quand il analyse les classes laborieuses comme classes dangereuses. On se retrouve dans des cas de figures où il n'existe pas finalement de mécanismes politiques d'intégration.

Didier Bigo : Je crois qu'il faut revenir sur la logique des fronts pour comprendre le double processus de désintégration et d'intégration. Il y a désintégration car les acteurs militarisés découpent le territoire national et s'approprient des zones territoriales en multipliant les luttes entre eux aux "frontières", mais en s'arrangeant pour conserver 
un niveau limité d'intensité de conflit : ce que j'appelais plus haut "homéopathisation de la guerre". Il y a intégration car chaque groupe militarisé cherche à reproduire à son profit la logique de contrôle étatique sur les populations désarmées en les taxant, en prélevant une partie des biens économiques et en taxant aussi les flux transnationaux des ONG ou des diasporas. Rémy a mis l'accent sur la question centrale pour l'avenir : logique politique ou réseaux mafieux? On pourrait penser à réutiliser le terme de prédation politique qu'emploie Jean-François Bayart...

Daniel Hermant : Dans le fond, on peut résumer la discussion en distinguant trois niveaux de conflit. Un niveau général et englobant : celui des grandes puissances, dont nous avons vu qu'elles ont imposé pendant très longtemps un cadre de lecture assez contraignant aux conflits. Un niveau intermédiaire où les puissances régionales (Pakistan, Inde, Iran, ou Chine, Vietnam, Thaïlande, etc.) jouent leurs intérêts politiques et stratégiques. L'affaiblissement des États provient souvent de ces ambitions exprimées en termes classiques de puissance. Un dernier niveau, qui est le niveau local des combattants. Ce niveau est relativement autonome des précédents et peut fonctionner pour rogner les pouvoirs de l'État.

Olivier Dabène: Dans le cas centraméricain, il existe un niveau régional bien spécifique. La régionalisation de l'explication des problèmes locaux permet aux gouvernements de trouver des sorties aux impasses dans lesquelles ils se sont enfermés. L'évolution de la situation centraméricaine est probablement moins négative que dans d'autres régions. Dans les évolutions prévisibles, l'Amérique centrale connaît sans doute plus de démocratie qu'au début de la décennie, mais également plus de violence. On voit apparaître la notion de démocratie violente...

31 Louis-Jean Duclos : On ne distingue pas assez entre conflit et violence politique ; l'un d'ordre public, hiérarchique et volontariste l'autre légitime ou délinquante, relevant de l'ordre social civil. Le premier renvoie à une notion de conflit stratégique ou à objectif(s) le second, à une sorte de bellicosité qu'on pourrait qualifier de structurelle. Les conflits dits stratégiques, dont l'archétype, non traité dans ce numéro, est le conflit israélo-arabe, met en scène deux acteurs ou groupes d'acteurs se disputant un enjeu ; la partie s'achève soit par dévalorisation, réelle ou imaginaire, de l'enjeu (le jeu n'en vaut plus la chandelle), soit par épuisement de I être ou de ressources de l'acteur (et le combat cessa faute de combattants). Parrni ces conflits stratégiques, on relèverait ici les conflits érythréo somali-éthiopiens (enjeu territorial), mais aussi, dans une certain mesure, le Cambodge, l'Afrique centrale, les États de l'Amérique centrale (appropriation de l'État-nation). Du moins sont-ils traité comme tels.

Dans les conflits du second type, structurels ou sociologiques, c'est l'organisation sociale elle-même, visitée par la modernité, qui serait polémogène. Le conflit résulterait ainsi, du moins en partie, de la "nature des choses". C'est à ce type d'explication qu'Olivier Roy semble recourir pour le cas afghan: une injection de modernité idéologique, financière et technique dans une société traditionnelle segmentaire et/ou clientélisée induit une conflictualité chronique et surtout médiatisable. Jadis, la guerre interclanique était le procédé auquel la société globale avait recours pour se réguler; la guerre civile d'aujourd'hui assume la même fonction de régulation sociale à laquelle il convient d'ajouter la correction des déséquilibres induits par la modernité. Autrement dit, cette modernité ferait obstacle au développement $\mathrm{du}$ processus de réconciliation préalable, selon le modèle 
"fonctionnaliste rénové" d'Apter, la modernisation politique. Qu'en penserait Bertrand Badie?

Également fonctionnaliste apparaît l'explication par Sélim Nasr de la crise libanaise, pour qui la guerre civile est essentiellement un "système social", celui qui permet aux chefs de guerre de maintenir leur pouvoir. Mais il y a déjà du micro-stratégique dans ce cas de figure. Il n'y a pas de cas purs : entre la "guerre d'état" (avec un petit é) et la guerre clausewitzienne à objectifs, un continuum permet de passer de l'une à l'autre.

"Rationnelle", alors, la seconde plus que la première? N'en croyons rien, répond Olivier Dabène, en tout cas pas sous sa forme prolongée, car celle-ci ne se conçoit que par la désobjectivation des enjeux, aussi propose-t-il fort opportunément un "délire guerrier", en quelque sorte, qui postule le désir de l'enjeu au-delà de la survie, ou l'absurde sacrifice de l'être en vue d'un plus ou mieux avoir. Il n'en reste pas moins qu'expliquer le conflit par la structure sociale et sa prolongation par la désobjectivation, c'est très bien. Cela n'explique cependant pas le pourquoi de la désobjectivation ni même le comment), ni le caractère belligène d'une structure sociale.

Didier Bigo : L'intérêt du numéro a été au contraire, me semble-t-il, d'insister sur les dynamiques de passage entre les deux formes que tu distingues. On ne peut pas séparer d'un côté les conflits stratégiques, militaires, et de l'autre les formes de violence dites internes. On a vu à quel point ces formes sont imbriquées aussi bien dans le conflit afghan que dans le cas de la corne de l'Afrique, du Cambodge ou du Liban. La prolongation des conflits fait souvent perdre la dimension stratégique et idéologique au profit de dimensions plus sociologiques de "guerre traditionnelle". Cela ne signifie pas une réduction de ces conflits à des dimensions tribales ou ethniques comme certains journalistes se dépêchent de l'écrire, participant à l'idéologie cartiériste du moment. Il y a plus sûrement un processus de politisation de l'ethnicité et non une ethnicisation du politique. A ce titre, le texte de Chapleau est très intéressant car il va à contrecourant des trajectoires éthiopiennes, somaliennes ou afghanes. En effet, les conflits idéologiques et syndicaux prennent le pas en Afrique du Sud sur les luttes provenant de la territorialisation (apartheid, homelands...). On se trouve dans une logique inverse du cas le plus commun, et notamment de celui des séparatismes. Cette situation a deux effets: la lutte devient politique au niveau global, mais, parallèlement, il y a atomisation de la violence. Ainsi, la population blanche, qui était jusqu'à présent à l'écart de la violence et qui ne l'envisageait que selon un modèle stratégique et interétatique, est brusquement en prise sur la violence et la criminalité, redécouvrant leurs significations politiques...

Daniel Hermant: Les dynamiques conflictuelles sont en effet importantes. Elles expliquent pour beaucoup la prolongation des conflits et répondent à certains mécanismes que Didier a montrés plus haut. Les transformations de l'ordre international sont aussi essentielles. Les conflits ne se dérouleront plus de la même manière ou, du moins, ne seront plus lus de la même manière car ils n'auront plus le même sens. La crise du Golfe risque de plus de modifier ce que nous venons de dire et de reposer les problèmes d'affrontements globaux des puissances régionales face aux puissances mondiales, de prolifération des armements nucléaires, chimiques, balistiques... Nous y reviendrons dans le prochain numéro. Merci à tous de votre participation à cette table ronde. 


\section{NOTES}

1. 1. Participants : Didier Bigo, Olivier Dabène, Louis-Jean Duclos, Alain Gascon, François Guilbert, Daniel Hermant, Zaki Laïdi, Rémy Leveau, Roland Marchal, Olivier Roy.

\section{RÉSUMÉS}

Illustrer, à travers ce choix de "conflits qui durent", l'originalité du projet qui nous réunit, à savoir : mieux comprendre les enracinements sociétaux des conflits dits locaux, voir que ces conflits, qui peuvent dans certaines occasions bouleverser les relations internationales, ont des causes internes puissantes, produisent des dynamiques sociales qui recomposent les sociétés et les cultures qui leur ont donné naissance, et constituent des phénomènes dont l'explication n'est jamais réductible, ni aux "politiques étrangères" des États, ni même à leurs géopolitiques, ou au degré de stabilité de "l'ordre international".

No summary

INDEX

Mots-clés : conflits, sociologie des conflits (polémologie) 